\title{
Screening, isolation, and decolonisation strategies in the control of meticillin resistant Staphylococcus aureus in intensive care units: cost effectiveness evaluation
}

\author{
(c) $\underset{0}{(1)(8)}$ OPEN ACCESS
}

\author{
Julie V Robotham mathematical modeller ${ }^{1}$, Nicholas Graves professor of health economics ${ }^{2}$, Barry \\ D Cookson director ${ }^{3}$, Adrian G Barnett associate professor ${ }^{2}$, Jennie A Wilson deputy director ${ }^{4}$, \\ Jonathan D Edgeworth consultant microbiologist and honorary senior lecturer ${ }^{56}$, Rahul Batra infection \\ research fellow ${ }^{6}$, Brian $\mathrm{H}$ Cuthbertson chief $^{7}$, Ben S Cooper senior research fellow ${ }^{89}$
}

${ }^{1}$ Modelling and Economics, Health Protection Agency, London NW9 5EQ, UK; ${ }^{2}$ Institute for Health and Biomedical Innovation, Queensland University of Technology, Brisbane, Australia; ${ }^{3}$ Laboratory of Healthcare Associated Infection, Health Protection Agency, London, UK; ${ }^{4}$ Infection Prevention and Control, Imperial College Healthcare NHS Trust, Hammersmith Hospital, London, UK; ${ }^{5}$ Department of Infectious Diseases, King's College London School of Medicine, Guy's Hospital, London, UK; ${ }^{6}$ Directorate of Infection, Guy's and St Thomas' NHS Foundation Trust, London, UK; ${ }^{7}$ Department of Critical Care Medicine, Sunnybrook Health Sciences Centre, Toronto, Canada; ${ }^{8}$ Mahidol-Oxford Tropical Medicine Research Unit, Faculty of Tropical Medicine, Mahidol University, Bangkok, Thailand ; ${ }^{9}$ Centre for Clinical Vaccinology and Tropical Medicine, Nuffield Department of Clinical Medicine, University of Oxford, Oxford, UK

\begin{abstract}
Objective To assess the cost effectiveness of screening, isolation, and decolonisation strategies in the control of meticillin resistant

Staphylococcus aureus (MRSA) in intensive care units.

Design Economic evaluation based on a dynamic transmission model. Setting England and Wales.

Population Theoretical population of patients on an intensive care unit. Main outcome measures Infections, deaths, costs, quality adjusted life years (QALYs), incremental cost effectiveness ratios for alternative strategies, and net monetary benefits.

Results All decolonisation strategies improved health outcomes and reduced costs. Although universal decolonisation (regardless of MRSA status) was the most cost effective in the short term, strategies using screening to target MRSA carriers may be preferred owing to the reduced risk of selecting for resistance. Among such targeted strategies, universal admission and weekly screening with polymerase chain reaction coupled with decolonisation using nasal mupirocin was the most cost effective.
\end{abstract}

This finding was robust to the size of intensive care units, prevalence of MRSA on admission, proportion of patients classified as high risk, and precise value of willingness to pay for health benefits. All strategies using isolation but not decolonisation improved health outcomes but costs were increased. When the prevalence of MRSA on admission to the intensive care unit was $5 \%$ and the willingness to pay per QALY gained was between $£ 20000$ ( $€ 23000 ; \$ 32000$ ) and $£ 30000$, the best such strategy was to isolate only those patients at high risk of carrying MRSA (either pre-emptively or after identification by admission and weekly screening for MRSA using chromogenic agar). Universal admission and weekly screening using polymerase chain reaction based detection of MRSA coupled with isolation was unlikely to be cost effective unless prevalence was high ( $10 \%$ of patients colonised with MRSA on admission).

Conclusions MRSA control strategies that use decolonisation are likely to be cost saving in an intensive care unit setting provided resistance is lacking, and combining universal screening using polymerase chain reaction with decolonisation is likely to represent good value for money if untargeted decolonisation is considered unacceptable. In intensive

Correspondence to: J V Robotham julie.robotham@hpa.org.uk

Extra material supplied by the author (see http://www.bmj.com/content/343/bmj.d5694/suppl/DC1)

Appendix 1: model simulating transmission of MRSA in intensive care unit setting

Appendix 2: estimation of parameters used in transmission model

Appendix 3: analysis without uncertainty, and scenario analyses

Table A2.1: values and sources of all parameters

Table A2.2: unadjusted daily probabilities of discharge and death

Table A2.3: unit costs in sterling

Table A3.1: evaluation of cost effectiveness frontier of screening and decolonisation policies

Table A3.2: evaluation of cost effectiveness frontier of screening and isolation policies 
care units where decolonisation is not implemented, evidence is insufficient to support universal screening for MRSA outside high prevalence settings.

\section{Introduction}

Despite the recent decline in incidence of meticillin resistant Staphylococcus aureus (MRSA) in several European countries, infection remains a major cause of avoidable morbidity and mortality in patients admitted to hospital, particularly those in intensive care units. MRSA infection increases the length of hospital stay, risk of death, and treatment costs. Patients may also become colonised with MRSA but remain asymptomatic. Such colonisation increases the risk of developing a clinical MRSA infection and is a source of cross infection.

Isolation and decolonisation are the two main targeted control measures for reducing the transmission of MRSA. Isolation interrupts cross infection through physical or behavioural barriers such as disposable gloves and aprons (contact precautions) or the placement of patients in isolation wards, single rooms, or cohort groups, with or without nursing staff designated for the exclusive care of MRSA infected patients. Decolonisation attempts to eliminate or suppress MRSA using topical antimicrobials such as chlorhexidine and intranasal mupirocin, thereby reducing the bacterial load available to cause endogenous infection and transmission to other patients. Isolation and decolonisation are often combined with screening to detect colonised patients. Early and accurate detection of colonised or infected patients allows timely implementation of interventions aimed at preventing transmission or infection. Many screening options exist, each with different performance characteristics: conventional culture, which is cheap but takes three or more days to give a result; chromogenic agars, which provide a result in one or two days; and polymerase chain reaction tests, which can give a result in a few hours and may be more sensitive but also more expensive than the other options. Which patients to screen and when also varies across settings: some screen all patients on admission and at regular intervals thereafter; some target screening to patients considered to be at high risk of carriage; and some do not screen at all.

Only a few of the many possible combinations of interventions have been examined in clinical trials, ${ }^{1-4}$ and economic evidence available to policy makers is scant. Consequently, control strategies vary from hospital to hospital. We evaluated the effectiveness and cost effectiveness of different combinations of screening, isolation, and decolonisation in intensive care units, where the consequences of MRSA acquisition are often greatest.

\section{Methods}

We developed a dynamic, stochastic, individual based model of MRSA transmission in an intensive care unit (fig $1 \Downarrow$ ), simulating the movement of patients, transmission process, and control interventions as well as associated patient outcomes (numbers of infections, discharges, and deaths). See web extra appendix 1 for further details.

\section{Interventions and screening scenarios}

We evaluated three screening technologies for MRSA: conventional culture, chromogenic agar, and polymerase chain reaction. Chromogenic agar yields results in 24 and 48 hours. Both times were considered, such that interventions based on a result at 24 hours could be amended after the result at 48 hours. For each technology we assessed three options for screening intensity: no screening (clinical cultures only), screening all patients on admission and weekly thereafter, and admission and weekly screening of patients considered to be at high risk of carrying MRSA. In all strategies we assumed clinical cultures were taken using the same technology as for screening (and conventional culture in the baseline strategy). Each of the screening strategies was combined with either isolation or decolonisation (table $1 \Downarrow$ ). In total we evaluated 12 strategies for screening plus isolation and nine for screening plus decolonisation. In both cases these included strategies applying interventions to patients identified as positive for MRSA through screening (or clinical specimens) in the intensive care unit and strategies applying interventions to all or all high risk intensive care unit admissions.

We considered isolation to be the application of contact precautions rather than physical separation, so capacity was unlimited. Isolation continued until three consecutive negative results from swabs were obtained. Decolonisation used nasal mupirocin administered three times daily (for strategies targeting MRSA carriers) or daily patient washings with chlorhexidine for five days (for strategies involving universal use).

We evaluated each strategy under baseline values for parameters (table $2 \Downarrow$ ), low and high prevalence settings ( $2 \%$ and $10 \%$ of patients colonised on admission), small and large intensive care units (five and 20 beds), and with $36 \%$ (rather than 18\%) of patients classified as high risk.

\section{Model parameters}

To account for uncertainty in the model parameters, we assigned probability distributions to each when appropriate (table 2). These probability distributions were derived from primary peer reviewed research articles where possible and from new analyses of primary data otherwise (see web extra table A2.1 and appendix 2). Neither approach was possible for some parameters concerning the impact of decolonisation treatment on transmission. Instead we considered two scenarios: a conservative baseline assumption that decolonisation treatment (with mupirocin) had no transmission blocking effect, and a "best guess" based on expert opinion (see web extra appendix 2). ${ }^{33}$

\section{Cost effectiveness evaluation}

Estimated costs included direct costs of the intervention, infection related treatment costs, and extra bed days and their associated opportunity costs (see web extra appendix 2).

Health benefits were reduced morbidity (during an episode on the intensive care unit) and reduced mortality owing to avoided infections, ${ }^{34}$ summarised by changes in quality adjusted life years (QALYs). ${ }^{35}$ From data on survival rates and preference based utility scores we estimated QALYs that accrued after hospital discharge (see web extra appendix 2). ${ }^{37}$

We used a time dependent model to estimate the additional length of stay and mortality attributable to MRSA infections, giving unadjusted daily discharge and death probabilities for patients with and without MRSA infection (see web extra table A2.2 and appendix 2). ${ }^{38}$ The daily relative risk ratio for discharge after infection was 0.60 (95\% confidence interval 0.53 to 0.68 ) and for death after infection was 1.19 (0.97 to 1.43 ).

The perspective for this analysis is that of a decision maker in healthcare who manages resources at a regional or national level. We aimed to represent the preferences of high level policy makers who seek to improve the economic efficiency of healthcare services. A shortcoming of this approach is that, unlike analyses from a wider societal perspective, costs incurred 
privately (such as out of pocket expenses) are excluded. However, recent studies show that these costs are likely to be small compared with hospital costs ${ }^{39} 40$ and are unlikely to change the conclusions from models.

We calculated the incremental cost effectiveness ratios, representing the cost per QALY gained on changing to an alternative intervention strategy (compared with the existing baseline strategy). Strategies were considered cost effective if they generated an incremental cost effectiveness ratio less than the usual National Health Service maximum willingness to pay of $£ 30000$ ( $€ 34000 ; \$ 48000$ ) per QALY ${ }^{41}$ We calculated incremental cost effectiveness ratios using baseline values for parameters (see web extra appendix 3 for scenario analyses).

We also calculated net monetary benefits, defined as $\Delta \mathrm{E} \times \lambda-\Delta \mathrm{C}$, where $\lambda$ is the willingness to pay per QALY gained, and $\Delta \mathrm{E}$ and $\Delta C$ are, respectively, changes in QALYs and costs compared with the baseline strategy. ${ }^{42}$ Probabilistic sensitivity analyses (accounting for uncertainty in all parameters) were carried out and used to calculate the expected net monetary benefit for each intervention (see web extra appendix 2).

\section{Results}

\section{Screening in combination with decolonisation}

All decolonisation strategies improved health outcomes and reduced costs (see web extra appendix 3, fig A3.1). This was because decolonisation, unlike isolation alone, can reduce the risk of infection in MRSA carriers. Universal decolonisation using chlorhexidine had a far higher probability of being cost effective than any other strategy (fig $2 \Downarrow$ ). At the willingness to pay threshold of $£ 30000$ per QALY, universal decolonisation using chlorhexidine had about a $70 \%$ chance of being the most cost effective strategy. The next best strategy—screening of all patients using polymerase chain reaction combined with mupirocin treatment of patients positive for MRSA — had about a 30\% probability of being cost effective. Universal decolonisation using chlorhexidine also provided the highest expected net monetary benefit at a willingness to pay of $£ 30$ 000 per QALY (fig 2). Pre-emptive decolonisation of all patients remained the most cost effective option in sensitivity analyses of varying intensive care unit size, prevalence of MRSA on admission, and proportion of high risk patients admitted (see web extra appendix 3).

Such blanket use of decolonisation agents may, however, hasten the emergence of resistance. Assuming that universal decolonisation using chlorhexidine is not a viable option, then the strategy of screening all patients using polymerase chain reaction and decolonising those identified as MRSA positive provides the highest expected net monetary benefit at all willingness to pay values over $£ 5000$ per QALY (fig 2). This result was not affected by the size of the intensive care unit, prevalence of MRSA on admission, or size of the high risk group (see web extra appendix 3). Results were also largely insensitive to assumptions about the effectiveness of decolonisation (see web extra appendix 3). However, if mupirocin was assumed to be completely ineffective at clearing MRSA carriage (although still able to suppress the risk of transmission and endogenous infection during treatment) then, after excluding universal decolonisation using chlorhexidine, screening all patients using polymerase chain reaction and decolonising those infected was fractionally over the usual cost effectiveness threshold, at $£ 30865$ per QALY gained (see web extra appendix 3 ).

\section{Screening in combination with isolation of patients}

Isolating only clinically identified cases resulted in the most unisolated bed days while MRSA positive (fig $3 \Downarrow$ ). Universal pre-emptive isolation minimised the number of unisolated days while MRSA positive but maximised inappropriate isolation (the isolation of MRSA negative patients). Limiting pre-emptive isolation to high risk patients decreased inappropriate isolation, but approximately halved appropriate isolation compared with universal pre-emptive isolation. Coupling pre-emptive isolation of high risk patients with subsequent amendment of isolation status based on the results of universal screening using conventional culture increased appropriate isolation and approximately halved the number of unisolated bed days while MRSA positive compared with limiting pre-emptive isolation to high risk patients. Among the different screening methodologies, screening using polymerase chain reaction led to the highest appropriate use of isolation. Targeting screening to high risk patients only slightly reduced appropriate isolation.

Strategies that rapidly isolate MRSA infected patients - universal pre-emptive isolation and isolation of MRSA positive patients identified using polymerase chain reaction-led to the greatest reduction in MRSA transmission and infection (fig $4 \Downarrow$ ), although differences in numbers of MRSA related infections and deaths were small (fig 4). However, even small differences in mortality can represent good value for money if the intervention cost is low.

Although all intervention strategies led to health gains compared with the strategy of doing nothing, in all cases the costs also increased (see web extra appendix 3, fig A3.2). Choosing between competing strategies requires consideration of cost effectiveness acceptability curves and the cost effectiveness acceptability frontier. Cost effectiveness acceptability curves show the probability of cost effectiveness dependent on the willingness to pay for health benefits. These revealed considerable uncertainty in the optimal screening and isolation strategy when the willingness to pay was within the usual NHS range of $£ 20000$ to $£ 30000$ (fig $5 \Downarrow$ ). No strategy had more than a $20 \%$ chance of being optimal.

Although cost effectiveness acceptability curves are valuable for representing the degree of uncertainty in an optimal strategy, they contain insufficient information for good decision making; identifying the strategy with the highest expected net monetary benefit is more useful. ${ }^{43}$ This is illustrated on the cost effectiveness acceptability frontier (fig 5), which shows the probability that the strategy with the highest expected net monetary benefit is cost effective for a given willingness to pay for health outcomes.

The cost effectiveness acceptability frontier representing screening and isolation strategies was split into five sections (fig 5). For a willingness to pay of up to about $£ 17000$ per QALY the approach to do nothing was favoured. Within the usual NHS willingness to pay range of $£ 20000$ to $£ 30000$ the greatest net monetary benefit would be provided by either a strategy of no screening but simply pre-emptive isolation of high risk patients or a strategy using chromogenic agar to screen high risk patients and subsequent isolation of those identified as MRSA positive. Screening all admissions using polymerase chain reaction became the optimum strategy only once the willingness to pay reached almost $£ 100000$ per QALY gained (over three times the usual NHS threshold).

At baseline values for parameters, a strategy of screening high risk patients with chromogenic agar (using both early and late results) was optimal. This remained true for smaller and larger 
intensive care units and a higher proportion of high risk patients. For a low prevalence setting ( $2 \%$ of patients colonised with MRSA on admission) screening high risk patients using chromogenic agar remained cost effective, but in a high prevalence setting (10\% colonised with MRSA on admission) screening all patients using polymerase chain reaction became cost effective (see web extra appendix 3).

\section{Discussion}

Although a model based analysis indicated that a strategy of universal topical decolonisation, regardless of MRSA status, was optimal in the short term, such untargeted use may select for resistance, making such strategies unsustainable in the long term. ${ }^{44}{ }^{45}$ For this reason, restricting the use of topical agents to patients known to be positive for MRSA may be preferred.

Excluding pre-emptive decolonisation of all patients, the optimal strategy was targeted decolonisation based on the outcomes of universal admission and weekly screens using polymerase chain reaction. The next best strategy was admission and weekly screening of all patients using chromogenic agar with decolonisation of those found to be MRSA positive (fig 2), although this had a much lower probability (compared with polymerase chain reaction) of being optimal at a $5 \%$ prevalence on admission.

Where decolonisation strategies are not used, admission and weekly screening coupled with isolation using contact precautions potentially reduces MRSA transmission, infections, and deaths. Use of polymerase chain reaction in this case, however, led to only modest reductions in MRSA infection rates compared with culture. This is a consequence of a small number of patients with unusually long lengths of stay accounting for a disproportionate number of MRSA related bed days. ${ }^{38}$ Although screening using polymerase chain reaction reduces the number of unisolated bed days while MRSA positive compared with culture, the percentage reduction will be small for patients with long stays.

Among strategies without decolonisation, universal polymerase chain reaction screening was unlikely to be cost effective within the usual NHS range of $£ 20000$ to $£ 30000$ per QALY gained, with chromogenic agar based screening and strategies targeting high risk patients being favoured. This reflects the lack of effect on progression from colonisation with MRSA to infection, and large uncertainty about the effect of contact precautions in reducing transmission, with a possibility that such precautions increase transmission. ${ }^{30}$ This could plausibly occur as a result of poorer compliance with hand hygiene associated with glove use. $^{46}$

\section{Strengths and weaknesses of the study}

Strengths of the modelling approach adopted here include the use of a dynamic transmission model to take into account that preventing MRSA acquisition in one patient benefits both that patient and other patients through reduced risk of cross infection. ${ }^{47-49}$ Such knock-on effects are important in high transmission settings such as intensive care units, and static decision analytical models would fail to take these effects into account. ${ }^{50-52}$ Accurate modelling of the length of stay distribution is also critical for assessing the benefits of rapid screening, as is accurately quantifying additional length of stay and mortality attributable to MRSA infections. ${ }^{38}$ These are likely to have been substantially over-estimated in the past by models that ignore the dynamic nature of infection..$^{53}$ Additionally, quantifying what is often substantial uncertainty in parameter values and accounting for it in strategy evaluations represents a more rational approach than one based only on point estimates for parameter values.

Limitations relate to simplifying assumptions and generalisability. Because the model ignores infections after discharge and because colonisation with MRSA may have longer term health consequences, as well as potentially leading to readmissions of colonised patients, the results presented are expected to underestimate health benefits and cost savings resulting from interventions to control MRSA.

For targeted strategies we used a decolonisation regimen of mupirocin alone, whereas many protocols may use skin antiseptics concurrently. As the best available evidence on effectiveness was for mupirocin alone, however, we used these to inform our model. Our estimates of the effectiveness of decolonisation are therefore likely to be conservative.

Pre-emptive universal decolonisation with agents such as chlorhexidine may reduce other healthcare associated infections, particularly catheter related ones, the benefit of which was not incorporated into our models. ${ }^{54-57}$ Conversely, decolonisation using nasal mupirocin has been reported to be associated with an increase of infections caused by other micro-organisms, although it remains unclear whether the link is causal. ${ }^{32}$ Therefore, with the possible exception of mupirocin based decolonisation interventions, cost effectiveness estimates reported here are likely to be conservative: true net monetary benefits are likely to be somewhat higher than those we report. Another assumption is lack of resistance to topical antimicrobial agents used for decontamination of colonised patients. This will not be the case for all settings; mupirocin resistance is not uncommon and there has been a recent report of an MRSA strain with a reduced susceptibility to chlorhexidine, ${ }^{44}$ the clinical relevance of which is still open to debate. ${ }^{58}{ }^{59}$ In view of this, in all evaluations of decolonisation regimens we have carried out a secondary analysis excluding universal use of decolonisation.

\section{Comparison with other studies}

A Health Technology Assessment of the cost effectiveness of MRSA screening in Scotland concluded that screening of all admissions to hospital using chromogenic agar with isolation of those identified as potential carriers proved most effective at reducing prevalence and most cost effective compared with no screening, and screening according to risk (of unit) and clinical risk assessment, regardless of whether such screening used agar, chromogenic agar, or polymerase chain reaction. ${ }^{60} \mathrm{~A}$ further study ${ }^{61}$ projected that universal screening for MRSA could result in an important reduction in MRSA rates over three to five years, and while chromogenic agar and polymerase chain reaction had a comparable effect, polymerase chain reaction was more costly.

Our study differed in that we limited the focus to intensive care units and we evaluated a wide range of intervention policies accounting for parameter uncertainty. We combined available evidence to determine distributions for parameters describing the effectiveness of interventions.

\section{Implications and future research}

Since 2006 the emphasis on the prevention and control of healthcare associated infections, particularly MRSA, has increased and this research sits in a context of wide ranging national guidance on infection control. ${ }^{62}{ }^{63}$ Although infection prevention methods of patient isolation and decolonisation (or suppression) are recommended, decisions remain locally driven and dependent on risk analysis. As a result, practices vary substantially between NHS trusts. While universal MRSA screening has been mandatory in England and Wales since 
December 2010, considerable uncertainty remains about the cost effectiveness of this and accompanying interventions. This study highlights the importance of evaluating the cost effectiveness of recommended prevention strategies and is especially important in supporting appropriate decision making during a period of cost restraint.

Our research found that even with conservative assumptions for the benefits of interventions, decolonisation strategies were highly likely to be cost effective in an intensive care unit setting and that, when combined with decolonisation, universal screening was likely to be a cost effective option. However, we highlight the need to be vigilant for the development of resistance to agents used for decolonisation. In contrast, we found no evidence to support universal screening (with any technology) as a cost effective intervention when accompanied by isolation. This reflects the lack of evidence on the effectiveness of contact precautions in substantially reducing transmission. Given this uncertainty, we found that isolation and screening strategies targeted to high risk patients were likely to be a more efficient use of resources.

The need for clinical investigations into the effectiveness of interventions has been further highlighted through this research. ${ }^{61}{ }^{64}$ The large extent of uncertainty about parameters reflects the scarcity of high quality research, and some important model parameters can only be estimated using single studies. Methodologically rigorous assessments from randomised trials of the effectiveness of interventions in reducing MRSA transmission and infections are rare. Considering a recent study, ${ }^{65}$ where large reductions $(62 \%)$ in MRSA infections were achieved using universal screening without accompanying decolonisation (among other interventions), rigorous evaluation of isolation should be a priority. Such studies would be of great value in helping to make better decisions about intervention policies.

Future work should also extend the model to describe long term patterns of patients' movements (between and within the hospital and community) and to account for the spread of resistance to topical antimicrobial agents. An increase in resistance in MRSA strains has the potential to greatly reduce the efficacy of decolonisation and therefore may severely compromise these strategies. Understanding the longer term consequences of widespread decolonisation, whether pre-emptive or targeted, and devising strategies to minimise the risks of emerging resistance should be a priority.

We thank John W Edmunds and Albert Jan van Hoek for useful discussions on the economic evaluation.

Contributors: BSC, NG, BDC, and JAW designed the study. JVR and BSC wrote the code, carried out the model parameterisation, computer simulations, and analysis, with economic input from NG and statistical input from $A G B$. RB, JDE, and BHC provided individual level intensive care unit data, and survival and quality of life data, as well as advice on analysis. JVR drafted and revised the manuscript with input from BSC, NG, AGB, BDC, JAW, JDE, RB, and BHC. All authors, external and internal, had full access to all of the data (including statistical reports and tables) in the study and can take responsibility for the integrity of the data and the accuracy of the data analysis. JVR, NG, and BSC act as the guarantors.

Funding: This work was funded by a grant from the Department of Health Policy Research Programme (PR-IP-0807-0410026). The views expressed are not necessarily those of the department. The authors' work was independent of the funders, who had no role in the study design, analysis of data, writing of the manuscript, or decision to submit for publication. We acknowledge support from the Mastering Hospital
Antimicrobial Resistance in Europe consortium. BSC acknowledges support from the Oak Foundation. The Mahidol Oxford Research Unit is supported by the Wellcome Trust.

Competing interests: All authors have completed the ICMJE uniform disclosure form at www.icmje.org/coi_disclosure.pdf (available on request from the corresponding author) and declare: no support from any organisation for the submitted work; no financial relationships with any organisations that might have an interest in the submitted work in the previous three years; no other relationships or activities that could appear to have influenced the submitted work.

Ethical approval: Not required.

Data sharing: Details of the computer code for the model are available from the corresponding author at Julie.Robotham@hpa.org.uk.

Cepeda JA, Whitehouse T, Cooper B, Hails J, Jones K, Kwaku F, et al. Isolation of patients in single rooms or cohorts to reduce spread of MRSA in intensive-care units: prospective two-centre study. Lancet 2005;365:295-304.

2 Jeyaratnam D, Whitty CJM, Phillips K, Liu D, Orezzi C, Ajoku U, et al. Impact of rapid screening tests on acquisition of meticillin resistant Staphylococcus aureus: cluster randomised crossover trial. BMJ 2008;336:927-30.

3 Bode LG, Kluytmans JA, Wertheim HF, Bogaers D, Vandenbroucke-Grauls CM, Roosendaal $R$, et al. Preventing surgical-site infections in nasal carriers of Staphylococcus aureus. N Engl J Med 2010;362:9-17.

4 Hardy K, Price C, Szczepura A, Gossain S, Davies R, Stallard N, et al. Reduction in the rate of methicillin-resistant Staphylococcus aureus acquisition in surgical wards by rapid screening for colonization: a prospective, cross-over study. Clin Microbiol Infect 2010;16:333-9.

5 Porter R, Subramani K, Thomas AN, Chadwick P. Nasal carriage of Staphylococcus aureus on admission to intensive care: incidence and prognostic significance. Intensive Care Med 2003;29:655-8.

6 Samad A, Banerjee D, Carbarns N, Ghosh S. Prevalence of methicillin-resistant Staphylococcus aureus colonization in surgical patients, on admission to a Welsh hospital. $J$ Hosp Infect 2002;51:43-6.

7 Theaker C, Ormond-Walshe S, Azadian B, Soni N. MRSA in the critically ill. J Hosp Infect 2001;48:98-102.

8 Thompson DS. Methicillin-resistant Staphylococcus aureus in a general intensive care unit. J R Soc Med 2004;97:521-6.

9 Dancer SJ, Coyne M, Speekenbrink A, Samavedam S, Kennedy J, Wallace PGM. MRSA acquisition in an intensive care unit. Am J Infect Control 2006;34:10-7.

10 Harbarth S, Sax H, Fankhauser-Rodriguez C, Schrenzel J, Agostinho A, Pittet D. Evaluating the probability of previously unknown carriage of MRSA at hospital admission. Am J Med 2006;119:15-23.

11 Eveillard M, Mortier E, Lancien E, Lescure F, Schmit J, Barnaud G, et al. Consideration of age at admission for selective screening to identify methicillin-resistant Staphylococcus aureus carriers to control dissemination in a medical ward. Am J Infect Control 2006;34:108-13

12 Safdar N, Narans L, Gordon B, Maki DG. Comparison of culture screening methods for detection of nasal carriage of methicillin-resistant Staphylococcus aureus: a prospective study comparing 32 methods. J Clin Microbiol 2003;41:3163-6.

13 Ben Nsira S, Dupuis M, Leclercq R. Evaluation of MRSA Select, a new chromogenic medium for the detection of nasal carriage of methicillin-resistant Staphylococcus aureus. Int $J$ Antimicrob Agents 2006;27:561-4.

14 Apfalter P, Assadian O, Kalczyk A, Lindenmann V, Makristathis A, Mustafa S, et al. Performance of a new chromogenic oxacillin resistance screen medium (Oxoid) in the detection and presumptive identification of methicillin-resistant Staphylococcus aureus. Diagn Microbiol Infect Dis 2002;44:209-11.

15 Lagacé-Wiens PR, Alfa MJ, Manickam K, Harding GK. Reductions in workload and reporting time by use of methicillin-resistant Staphylococcus aureus screening with MRSASelect medium compared to mannitol-salt medium supplemented with oxacillin. $J$ Clin Microbiol 2008;46:1174-7.

16 Cunningham R, Jenks P, Northwood J, Wallis M, Ferguson S, Hunt S. Effect on MRSA transmission of rapid PCR testing of patients admitted to critical care. J Hosp Infect 2007:65:24-8.

17 Harbarth S, Masuet-Aumatell C, Schrenzel J, Francois P, Akakpo C, Renzi G, et al. Evaluation of rapid screening and pre-emptive contact isolation for detecting and controlling methicillin-resistant Staphylococcus aureus in critical care: an interventional cohort study. Crit Care 2006;10:R25.

18 Nahimana I, Francioli P, Blanc DS. Evaluation of three chromogenic media (MRSA-ID, MRSA-Select and CHROMagar MRSA) and ORSAB for surveillance cultures of methicillin-resistant Staphylococcus aureus. Clin Microbiol Infect 2006;12:1168-74.

19 Van Hal SJ, Stark D, Lockwood B, Marriott D, Harkness J. Methicillin-resistant Staphylococcus aureus (MRSA) detection: comparison of two molecular methods (IDI-MRSA PCR assay and GenoType MRSA Direct PCR assay) with three selective MRSA agars (MRSA ID, MRSASelect, and CHROMagar MRSA) for use with infection-control swabs. J Clin Microbiol 2007;45:2486-90.

20 Perry JD, Davies A, Butterworth LA, Hopley ALJ, Nicholson A, Gould FK. Development and evaluation of a chromogenic agar medium for methicillin-resistant Staphylococcus aureus. J Clin Microbiol 2004;42:4519-23.

21 Compernolle V, Verschraegen G, Claeys G. Combined use of Pastorex Staph-Plus and either of two new chromogenic agars, MRSA ID and CHROMagar MRSA, for detection of methicillin-resistant Staphylococcus aureus. J Clin Microbiol 2007;45:154-8.

22 Van Hal SJ, Jennings Z, Stark D, Marriott D, Harkness J. MRSA detection: comparison of two molecular methods (BD GeneOhm PCR assay and Easy-Plex) with two selective MRSA agars (MRSA-ID and Oxoid MRSA) for nasal swabs. Eur $\mathrm{J}$ Clin Microbiol Infect Dis 2009;28:47-53.

23 Zhang SX, Drews SJ, Tomassi J, Katz KC. Comparison of two versions of the IDI-MRSA assay using charcoal swabs for prospective nasal and nonnasal surveillance samples. $J$ Clin Microbiol 2007;45:2278-80. 


\section{What is already known on this topic}

Meticillin resistant Staphylococcus aureus (MRSA) continues to cause a high burden of disease in intensive care units (ICUs)

Many ICUs use MRSA screening combined with isolation or decolonisation in an attempt to reduce MRSA infections, but practices vary widely between units

New screening technologies allow MRSA carriage to be detected more rapidly and with greater sensitivity, although at greater cost than conventional approaches

\section{What this study adds}

Screening and decolonisation are potentially cost saving in ICUs if MRSA is sensitive to decolonising agents

Universal polymerase chain reaction based screening accompanied by decolonisation is likely to represent an efficient use of resources

Targeting screening and isolation to high risk groups is likely to be a more efficient use of resources than universal screening with isolation or universal pre-emptive isolation

24 Keshtgar MRS, Khalili A, Coen PG, Carder C, Macrae B, Jeanes A, et al. Impact of rapid molecular screening for meticillin-resistant Staphylococcus aureus in surgical wards. $\mathrm{Br}$ $J$ Surg 2008;95:381-6.

25 Huletsky A, Lebel P, Picard FJ, Bernier M, Gagnon M, Boucher N, et al. Identification of methicillin-resistant Staphylococcus aureus carriage in less than 1 hour during a hospital surveillance program. Clin Infect Dis 2005;40:976-81.

26 Rossney AS, Herra CM, Fitzgibbon MM, Morgan PM, Lawrence MJ, O'Connell B. Evaluation of the IDI-MRSA assay on the SmartCycler real-time PCR platform for rapid detection of MRSA from screening specimens. Eur J Clin Microbiol Infect Dis 2007;26:459-66.

27 Warren DK, Guth RM, Coopersmith CM, Merz LR, Zack JE, Fraser VJ. Impact of a methicillin-resistant Staphylococcus aureus active surveillance program on contact precaution utilization in a surgical intensive care unit. Crit Care Med 2007;35:430-4

28 Paule SM, Hacek DM, Kufner B, Truchon K, Thomson RBJ, Kaul KL, et al. Performance of the BD GeneOhm methicillin-resistant Staphylococcus aureus test before and during high-volume clinical use. J Clin Microbiol 2007;45:2993-8.

29 Bishop EJ, Grabsch EA, Ballard SA, Mayall B, Xie S, Martin R, et al. Concurrent analysis of nose and groin swab specimens by the IDI-MRSA PCR assay is comparable to analysis by individual-specimen PCR and routine culture assays for detection of colonization by methicillin-resistant Staphylococcus aureus. J Clin Microbiol 2006;44:2904-8.

30 Kypraios T, O'Neill PD, Huang SS, Rifas-Shiman SL, Cooper BS. Assessing the role of undetected colonization and isolation precautions in reducing methicillin-resistant Staphylococcus aureus transmission in intensive care units. BMC Infect Dis 2010;10:29

31 Rohr U, Mueller C, Wilhelm M, Muhr G, Gatermann S. Methicillin-resistant Staphylococcus aureus wholebody decolonisation among hospitalized patients with variable site colonisation by using mupirocin in combination with octenidine dihydrochloride. J Hosp Infect 2003:54:305-9.

32 Van Rijen M, Bonten M, Wenzel R, Kluytmans J. Mupirocin ointment for preventing Staphylococcus aureus infections in nasal carriers. Cochrane Database Syst Rev 2008;8:CD006216.

33 O'Hagan A, Buck C, Daneshkhah A, Eiser JR, Garthwaite PH, Jenkinson D, et al. Uncertain judgements: eliciting experts' probabilities. Wiley, 2006.

34 Cosgrove SE, Qi Y, Kaye KS, Harbarth S, Karchmer AW, Carmeli Y. The impact of methicillin resistance in Staphylococcus aureus bacteremia on patient outcomes: mortality, length of stay, and hospital charges. Infect Control Hosp Epidemiol 2005;26:166-74.

35 Graves N, Halton K, Lairson D. Economics and preventing hospital-acquired infection—broadening the perspective. Infect Control Hosp Epidemiol 2007;28:178-84.

36 Graves N. Economics and preventing hospital-acquired infection. Emerg Infect Dis 2004;10:561-6.

37 Cuthbertson BH, Roughton S, Jenkinson D, Maclennan G, Vale L. Quality of life in the five years after intensive care: a cohort study. Crit Care 2010;14:R6.

38 Barnett AG, Batra R, Graves N, Edgeworth J, Robotham J, Cooper B. Using a longitudinal model to estimate the effect of methicillin-resistant Staphylococcus aureus infection on length of stay in an intensive care unit. Am J Epidemiol 2009;170:1186-94.

39 Graves N, Halton K, Curtis M, Doidge S, Lairson D, McLaws M, et al. Costs of surgical site infection that appear after discharge. Emerg Infect Dis 2006;12:831-5.

40 Graves N, Halton K, Doidge S, Clements A, Lairson D, Whitby M. Who bears the cost of healthcare-acquired surgical site infection? J Hosp Infect 2008;69:274-82.

41 Rawlins MD, Culyer AJ. National Institute for Clinical Excellence and its value judgments. BMJ 2004;329:224-7.

42 Claxton K, Sculpher M, McCabe C, Briggs A, Akehurst R, Buxton M, et al. Probabilistic sensitivity analysis for NICE technology assessment: not an optional extra. Health Econ 2005;14:339-47.

43 Barton G, Briggs A, Fenwick E. Optimal cost-effectiveness decisions: the role of the cost-effectiveness acceptability curve (CEAC), the cost-effectiveness acceptability frontie (CEAF), and the expected value of perfect information (EVPI). Value Health 2008;11:886-97.

44 Batra R, Cooper BS, Whiteley C, Patel AK, Wyncoll D, Edgeworth JD. Efficacy and limitation of a chlorhexidine-based decolonization strategy in preventing transmission of methicillin-resistant Staphylococcus aureus in an intensive care unit. Clin Infect Dis 2010;50:210-7.

45 Cookson BD. The emergence of mupirocin resistance: a challenge to infection control and antibiotic prescribing practice. J Antimicrob Chemother 1998;41:11-8.

46 Girou E, Chai SH, Oppein F, Legrand P, Ducellier D, Cizeau F, et al. Misuse of gloves: the foundation for poor compliance with hand-hygiene and potential for microbial transmission? J Hosp Infect 2004;57:162-9.
47 Roberts T, Robinson S, Barton P, Bryan S, McCarthy A, Macleod J, et al. The correct approach to modelling and evaluating chlamydia screening. Sex Transm Infect 2004:80:324-5.

48 De Vries R, Kretzschmar M, Schellekens JF, Versteegh FG, Westra TA, Roord JJ, et al. Cost-effectiveness of adolescent pertussis vaccination for the Netherlands: using an individual-based dynamic model. PLOS One 2010;5:e13392.

49 Turner K, Adams E, Grant A, Macleod J, Bell G, Clarke J, et al. Costs and cost effectiveness of different strategies for chlamydia screening and partner notification: an economic and mathematical modelling study. BMJ 2011;342:c7250.

50 Lee BY, Wiringa AE, Bailey RR, Goyal V, Tsui B, Lewis GJ, et al. The economic effect of screening orthopedic surgery patients preoperatively for methicillin-resistant Staphylococcus aureus. Infect Control Hosp Epidemiol 2010;31:1130-8.

51 Nelson RE, Samore MH, Smith KJ, Harbarth S, Rubin MA. Cost-effectiveness of adding decolonization to a surveillance strategy of screening and isolation for methicillin-resistant Staphylococcus aureus carriers. Clin Microbiol Infect 2010;16:1740-6.

52 Brown J, Paladino JA. Impact of rapid methicillin-resistant Staphylococcus aureus polymerase chain reaction testing on mortality and cost effectiveness in hospitalized patients with bacteraemia: a decision model. Pharmacoeconomics 2010;28:567-75.

53 Beyersmann J, Gastmeier P, Wolkewitz M, Schumacher M. An easy mathematical proof showed that time-dependent bias inevitably leads to biased effect estimation. J Clin Epidemiol 2008:61:1216-21.

54 Bleasdale SC, Trick WE, Gonzalez IM, Lyles RD, Hayden MK, Weinstein RA. Effectiveness of chlorhexidine bathing to reduce catheter-associated bloodstream infections in medical intensive care unit patients. Arch Intern Med 2007;167:2073-9.

55 Evans HL, Dellit TH, Chan J, Nathens AB, Maier RV, Cuschieri J. Effect of chlorhexidine whole-body bathing on hospital-acquired infections among trauma patients. Arch Surg 2010;145:240-6.

56 Popovich KJ, Hota B, Hayes R, Weinstein RA, Hayden MK. Effectiveness of routine patient cleansing with chlorhexidine gluconate for infection prevention in the medical intensive care unit. Infect Control Hosp Epidemiol 2009;30:959-63.

57 Climo MW, Sepkowitz KA, Zuccotti G, Fraser VJ, Warren DK, Perl TM, et al. The effect of daily bathing with chlorhexidine on the acquisition of methicillin-resistant Staphylococcus aureus, vancomycin-resistant Enterococcus, and healthcare-associated bloodstream infections: results of a quasi-experimental multicenter trial. Crit Care Med 2009;37:1858-65.

58 Lee AS, Macedo-Vinas M, Francois P, Renzi G, Schrenzel J, Vernaz N, et al. Impact of combined low-level mupirocin and genotypic chlorhexidine resistance on persistent methicillin-resistant Staphylococcus aureus carriage after decolonization therapy: case-control study. Clin Infect Dis 2011:52:1422-30.

59 Meyer B, Cookson B. Does microbial resistance or adaptation to biocides create a hazard in infection prevention and control? J Hosp Infect 2010;76:200-5.

60 Ritchie K, Bradbury I, Eastgate J, Foster L, Iqbal K, MacPherson K, et al. Consultation report on health technology: clinical and cost effectiveness of screening for MRSA. NHS Quality Improvement Scotland, 2006

61 Health Protection Scotland. Pathfinder implementation study. 2011. www.hps.scot.nhs. uk/haiic/sshaip/mrsascreeningprogramme.aspx.

62 Coia JE, Duckworth GJ, Edwards DI, Farrington M, Fry C, Humphreys H, et al. Guidelines for the control and prevention of meticillin-resistant Staphylococcus aureus (MRSA) in healthcare facilities. J Hosp Infect 2006;63:S1-44.

63 National Health Service. Clean, safe care: reducing MRSA and other healthcare associated infections. Department of Health. 2011. www.clean-safe-care.nhs.uk.

64 Cooper BS, Stone SP, Kibbler CC, Cookson BD, Roberts JA, Medley GF, et al. Isolation measures in the hospital management of methicillin resistant Staphylococcusaureus (MRSA): systematic review of the literature. BMJ 2004;329:533.

65 Jain R, Kralovic SM, Evans ME, Ambrose M, Simbartl LA, Obrosky DS, et al. Veterans Affairs initiative to prevent methicillin-resistant Staphylococcus aureus infections. N Engl $J$ Med 2011;364:1419-30.

Accepted: 9 August 2011

\section{Cite this as: BMJ 2011;343:d5694}

This is an open-access article distributed under the terms of the Creative Commons Attribution Non-commercial License, which permits use, distribution, and reproduction in any medium, provided the original work is properly cited, the use is non commercial and is otherwise in compliance with the license. See: http://creativecommons.org/licenses/by$\mathrm{nc} / 2.0 /$ and http://creativecommons.org/licenses/by-nc/2.0/legalcode. 


\section{Tables}

Table 1 | Combinations of screening and interventions for the control of meticillin resistant Staphylococcus aureus (MRSA) in patients on intensive care units

\begin{tabular}{|c|c|c|c|c|}
\hline \multirow[b]{2}{*}{ Screening } & \multirow[b]{2}{*}{ Screening method } & \multicolumn{3}{|c|}{ Intervention } \\
\hline & & Patient group* & Timing & Procedure \\
\hline No screening & $\begin{array}{l}\text { Conventional culture of clinical } \\
\text { swabs only }\end{array}$ & MRSA positive & On result & $\begin{array}{l}\text { Isolation or decolonisation } \\
\text { (chlorhexidine) }\end{array}$ \\
\hline No screening & $\begin{array}{l}\text { Conventional culture of clinical } \\
\text { swabs only }\end{array}$ & All patients & Pre-emptively & $\begin{array}{l}\text { Isolation or decolonisation } \\
\text { (chlorhexidine) }\end{array}$ \\
\hline No screening & $\begin{array}{l}\text { Conventional culture of clinical } \\
\text { swabs only }\end{array}$ & High risk patients & Pre-emptively & $\begin{array}{l}\text { Isolation or decolonisation } \\
\text { (chlorhexidine) }\end{array}$ \\
\hline All patients & Conventional culture & High risk patients & $\begin{array}{l}\text { Pre-emptively (amended on } \\
\text { screen result) }\end{array}$ & Isolation† \\
\hline \multirow{4}{*}{$\begin{array}{l}\text { All patients on } \\
\text { admission, and } \\
\text { weekly thereafter }\end{array}$} & Conventional culture & MRSA positive & On result & Isolation or decolonisation (mupirocin) \\
\hline & Chromogenic agar & MRSA positive & On 48 hour result & Isolation or decolonisation (mupirocin) \\
\hline & & MRSA positive & $\begin{array}{l}\text { On } 24 \text { hour result (amended on } \\
48 \text { hour result) }\end{array}$ & Isolationt \\
\hline & Polymerase chain reaction & MRSA positive & On result & Isolation or decolonisation (mupirocin) \\
\hline \multirow{4}{*}{$\begin{array}{l}\text { High risk patients on } \\
\text { admission, and } \\
\text { weekly thereafter }\end{array}$} & Conventional culture & MRSA positive & On result & Isolation or decolonisation (mupirocin) \\
\hline & Chromogenic agar & MRSA positive & On 48 hour result & Isolation or decolonisation (mupirocin) \\
\hline & & MRSA positive & $\begin{array}{l}\text { On } 24 \text { hour result (amended on } \\
48 \text { hour result) }\end{array}$ & Isolationt \\
\hline & Polymerase chain reaction & MRSA positive & On result & Isolation or decolonisation (mupirocin) \\
\hline
\end{tabular}

In all strategies, if MRSA was recovered from a clinical specimen the patient was isolated or decolonised according to the specified intervention. *MRSA positive patients are believed to be colonised or infected with MRSA based on clinical or screening swab result.

†Decolonisation not applicable. 
Table 2| Values and sources of parameters. Values are means (standard deviations) or [values for sensitivity analyses] unless stated otherwise

\begin{tabular}{|c|c|c|}
\hline Parameters & Values & Sources \\
\hline \multicolumn{3}{|l|}{ Population } \\
\hline Prevalence of MRSA on admission & $0.05[0.02$ and 0.10$]$ & $1,5-9$ \\
\hline Prevalence of MRSA in high risk patients on admission & 2.4×prevalence of MRSA on admission & 10,11 \\
\hline Proportion of high risk patients & $0.18[0.36]$ & 10,11 \\
\hline No of intensive care unit beds & $10[5$ and 20$]$ & ICNARC \\
\hline \multicolumn{3}{|l|}{ Transmission } \\
\hline Daily probability of cross colonisation per source ${ }^{*}$ & $0.0037(0.00043)$ & \multirow{3}{*}{$\begin{array}{l}\text { Analysis of individual data (see web extra } \\
\text { appendix 2) }\end{array}$} \\
\hline Daily probability of cross infection per source* & $0.0006(0.00023)$ & \\
\hline Daily probability of progression from colonisation to infection* & $0.047(0.0094)$ & \\
\hline \multicolumn{3}{|l|}{ Screening } \\
\hline \multicolumn{3}{|l|}{ Conventional culturet: } \\
\hline Sensitivity (\%)‡ & $68.15(19.39)$ & \multirow[t]{2}{*}{$12-14$} \\
\hline Specificity (\%)‡ & $88.23(6.33)$ & \\
\hline Turnaround time (days)§ & 4 & $15-17$ \\
\hline \multicolumn{3}{|l|}{ Chromogenic agar (48 hour result): } \\
\hline Sensitivity (\%)‡ & $82.55(4.27)$ & \multirow[t]{2}{*}{$18-22$} \\
\hline Specificity (\%)‡ & $83.05(17.72)$ & \\
\hline Turnaround time (days)§ & 3 & $15,17,18,20$ \\
\hline \multicolumn{3}{|l|}{ Chromogenic agar (24 hour result): } \\
\hline Sensitivity $(\%)^{*}$ & $62.17(12.49)$ & \multirow[t]{2}{*}{$13,18,20-22$} \\
\hline Specificity $(\%)^{*}$ & $97.13(4.17)$ & \\
\hline Turnaround time (days)§ & 2 & 17 \\
\hline \multicolumn{3}{|l|}{ Polymerase chain reaction: } \\
\hline Sensitivity (\%)‡ & $88.40(5.10)$ & \multirow[t]{2}{*}{$16,19,22-29$} \\
\hline Specificity (\%)‡ & $83.8(4.74)$ & \\
\hline Turnaround time (days)§ & 1 & $16,17,19,24,26$ \\
\hline \multicolumn{3}{|l|}{ Isolation } \\
\hline Transmissibility of patient undergoing isolation precautions (\% reduction) & $36.5(62.2)$ & 30 \\
\hline \multicolumn{3}{|l|}{ Decolonisation } \\
\hline Treated patients who reverted to MRSA negative state (\%) & $53[0$ and 100$]$ & 31 [assumptions] \\
\hline \multicolumn{3}{|l|}{ Effect on susceptibility to colonisation (\% reduction): } \\
\hline Bodywash (chlorhexidine) & $65(7)$ & Analysis of individual data \\
\hline Topical therapy (mupirocin) & 0 [33] & Assumption [ๆ] \\
\hline \multicolumn{3}{|l|}{ Effect on susceptibility to infection (\% reduction): } \\
\hline Bodywash (chlorhexidine) & $66(21)$ & Analysis of individual data \\
\hline Topical therapy (mupirocin) & $0[10]$ & Assumption [ף] \\
\hline \multicolumn{3}{|l|}{ Effect on transmissibility of infectious patient (\% reduction): } \\
\hline Topical therapy (mupirocin) & $0[12.5]$ & Assumption [ף] \\
\hline \multicolumn{3}{|l|}{$\%$ reduction in probability of progression or self infection: } \\
\hline Bodywash (chlorhexidine) & $31(18)$ & Analysis of individual data \\
\hline Topical therapy (mupirocin) & $33(12)[47]$ & 32 [П] \\
\hline
\end{tabular}

ICNARC=Intensive Care National Audit and Research Centre.

For sensitivity analysis all values expressed as percentages are assumed to follow normal distributions, truncated at $100 \%$.

*Probability of transmission from single MRSA source to single susceptible patient on same ward.

†Medium containing mannitol, salt, and oxacillin.

‡Arithmetic mean (standard deviation) from listed source.

$\S$ Means of reported laboratory processing times, adjusted to account for ward delays as estimated in Harbarth et al 2006. ${ }^{17}$

ףEstimates obtained from formal elicitation of expert opinion (see web extra appendix 2 for methods). 


\section{Figures}

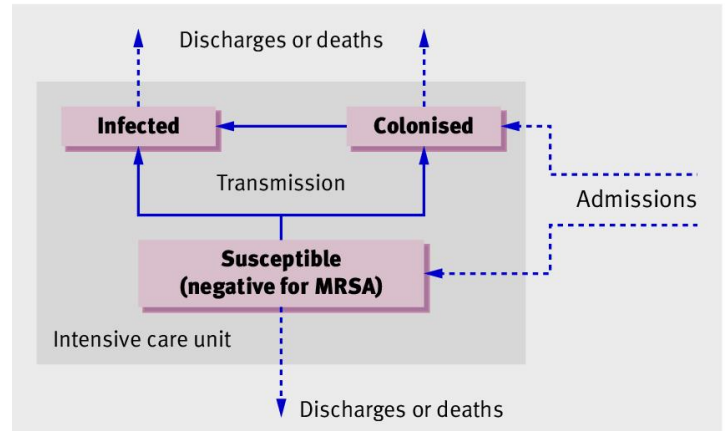

Fig 1 Model schematic showing possible movements of patients (dotted arrows) and transitions between states (solid arrows). MRSA=meticillin resistant Staphylococcus aureus 
- Do nothing (decolonisation with chlorhexidine of clinical cases only)

- - - Universal pre-emptive decolonisation (with chlorhexidine)

----- Pre-emptive decolonisation of high risk patients (with chlorhexidine)

Universal screening+decolonisation of MRSA positive patients (with mupirocin)

- - Conventional culture+decolonisation

- Chromogenic agar+decolonisation

---- Polymerase chain reaction+decolonisation

Screening of high risk patients+decolonisation of MRSA

positive patients (with mupirocin)

- - - Conventional culture+decolonisation

....-. Chromogenic agar+decolonisation

- - Polymerase chain reaction+decolonisation
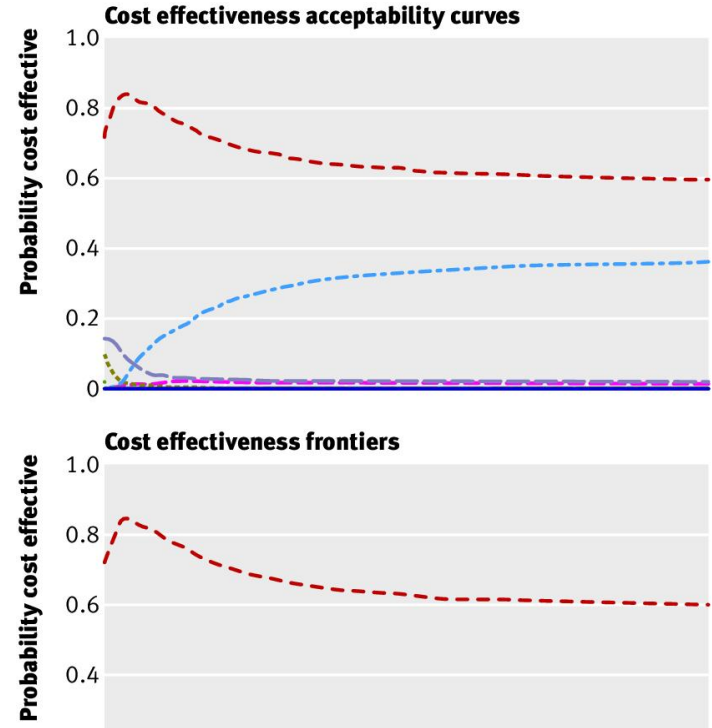

0.2

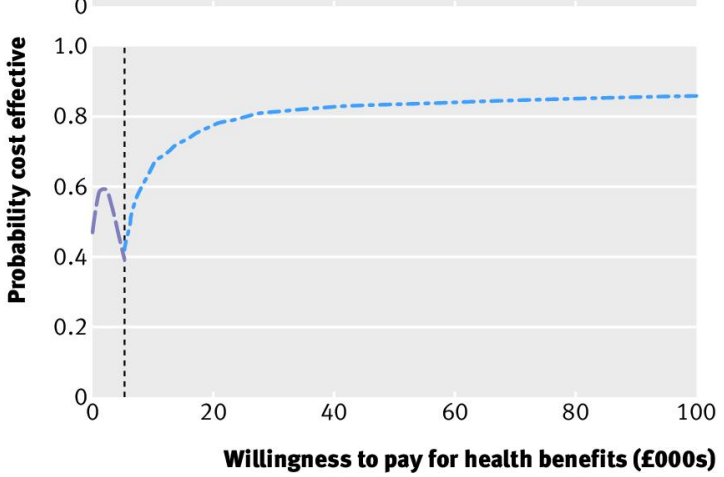

Fig 2 Cost effectiveness acceptability curves and frontiers for screening and decolonisation strategies. Each line on cost effectiveness acceptability curve represents the proportion of simulations for a particular strategy that are cost effective, under a range of values for willingness to pay for health benefits. Lines in cost effectiveness acceptability frontiers depict scenarios with highest expected net monetary benefit, dependent on willingness to pay for health benefits. The first frontier compares all strategies; the second excludes universal decolonisation 
Do nothing (isolation of clinical cases only) Pre-emptive isolation

$\square$ Universal pre-emptive isolation

$\square$ Pre-emptive isolation of high risk patients

$\square$ Pre-emptive isolation (+amendment on results of conventional culture)

Universal screening+isolation of MRSA positive patients

$\square$ Conventional culture+isolation

$\square$ Chromogenic agar+isolation

$\mathbf{D}$ Chromogenic agar (24 and 48 hour result)+isolation

Polymerase chain reaction+isolation

Screening of high risk patients+isolation of MRSA positive patients

Conventional culture+isolation

Chromogenic agar+isolation

Chromogenic agar (24 and 48 hour result)+isolation

Solymerase chain reaction+isolation
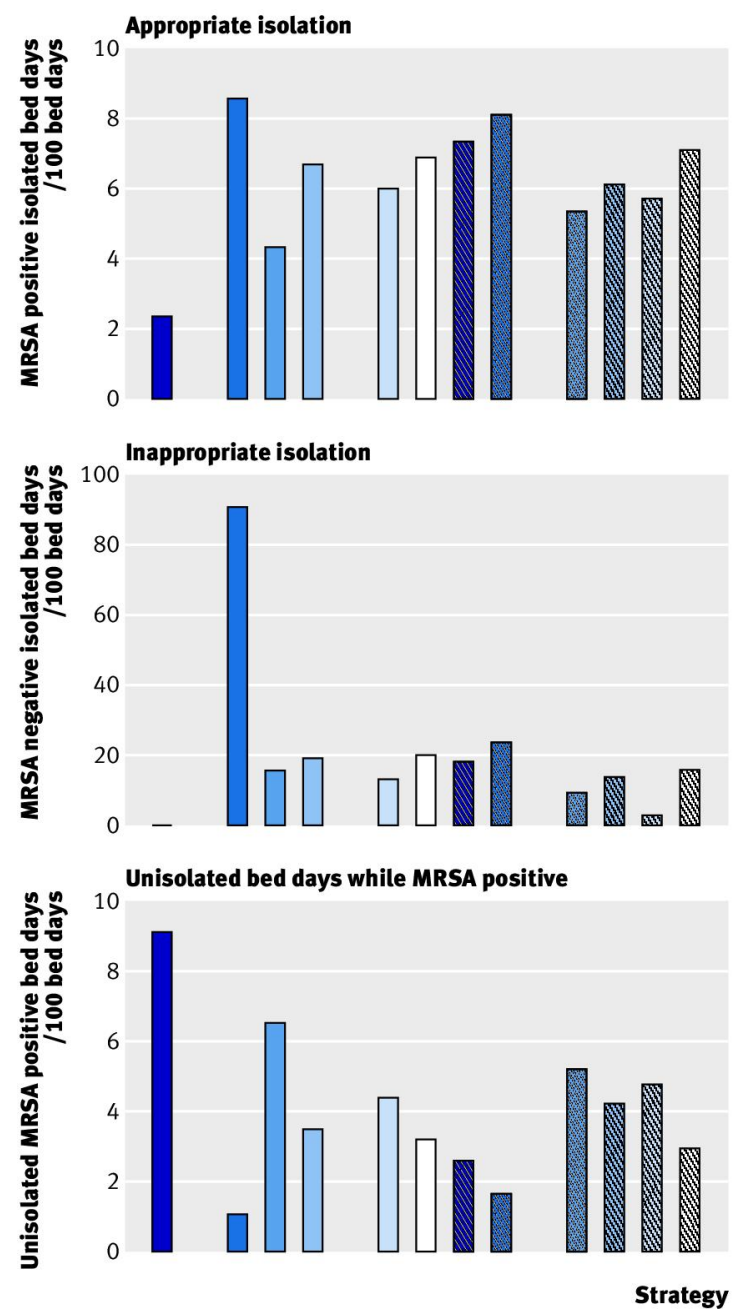

Fig 3 Use of isolation under each screening and isolation strategy, showing appropriate isolation (isolation of MRSA positive patients), inappropriate isolation (isolation of MRSA negative patients), and bed days spent unisolated while MRSA positive 
Do nothing (isolation of clinical cases only) Pre-emptive isolation

$\square$ Universal pre-emptive isolation

$\square$ Pre-emptive isolation of high risk patients

$\square$ Pre-emptive isolation (+amendment on results of conventional culture)

Universal screening+isolation of MRSA positive patients

$\square$ Conventional culture+isolation

$\square$ Chromogenic agar+isolation

$\mathbf{D}$ Chromogenic agar (24 and 48 hour result)+isolation

Polymerase chain reaction+isolation

Screening of high risk patients+isolation of MRSA positive patients

Conventional culture+isolation

Chromogenic agar+isolation

Chromogenic agar (24 and 48 hour result)+isolation

Solymerase chain reaction+isolation
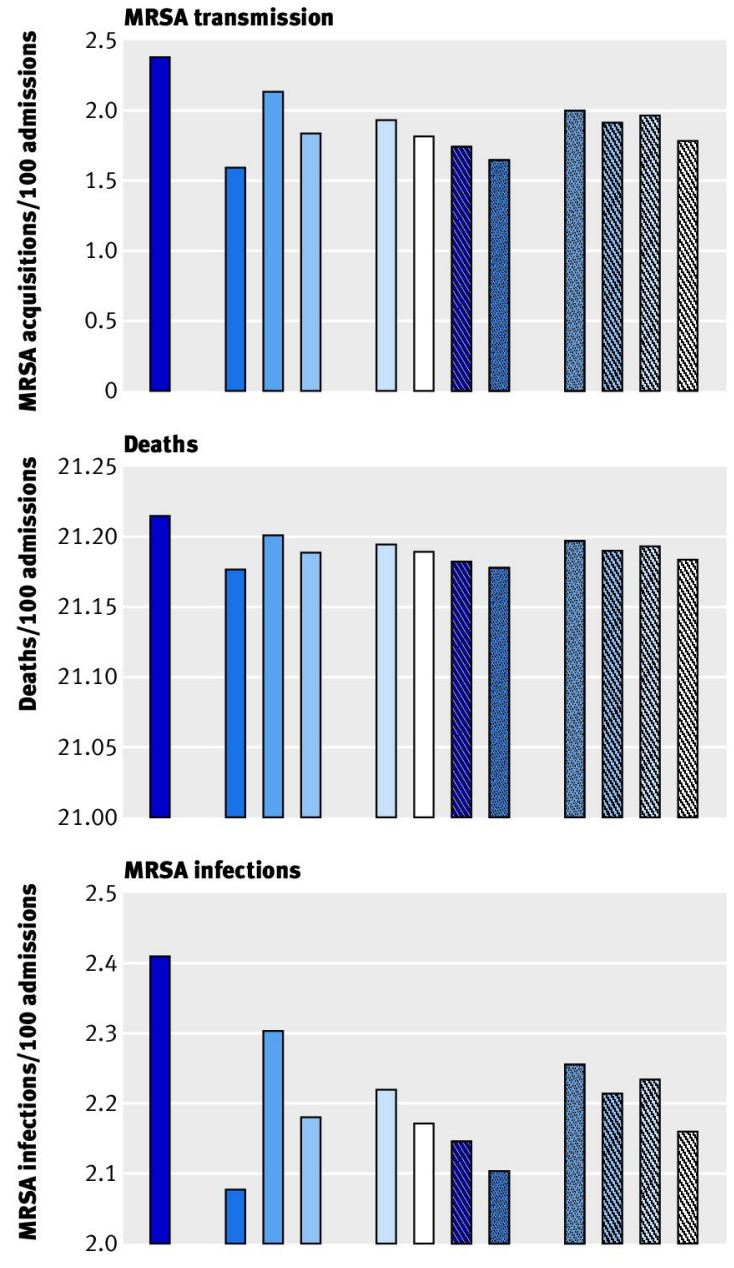

Strategy

Fig 4 Patient outcomes under each screening and isolation strategy, showing new acquisitions (transmissions) of MRSA by patients in an intensive care unit, total number of MRSA infections in an intensive care unit, and total number of deaths (all per 100 admissions) 
Do nothing (isolation of clinical cases only)

\section{Pre-emptive isolation}

- - - Universal pre-emptive isolation

----- Pre-emptive isolation of high risk patients

-.- Pre-emptive isolation (+amendment on results of conventional culture)

Universal screening+isolation of MRSA positive patients

- - Conventional culture+isolation

- Chromogenic agar+isolation

... Chromogenic agar (24 and 48 hour result)+isolation

---- Polymerase chain reaction+isolation

Screening of high risk patients+isolation of MRSA positive patients

- - Conventional culture+isolation

....-. Chromogenic agar+isolation

--- Chromogenic agar (24 and 48 hour result)+isolation

-- Polymerase chain reaction+isolation
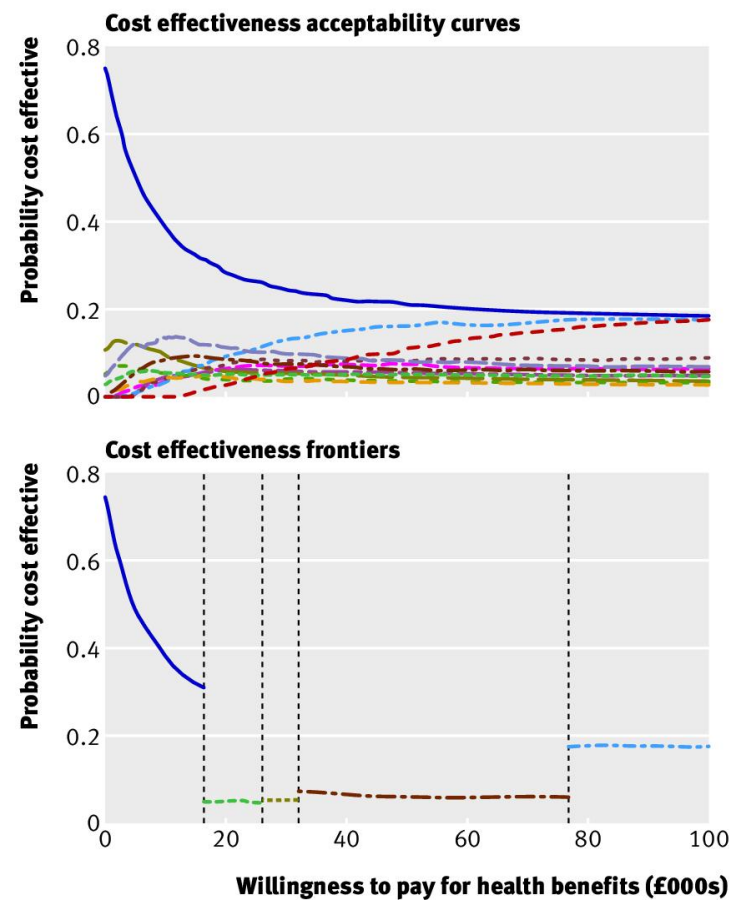

Fig 5 Cost effectiveness acceptability curves and frontier for screening and isolation strategies. Lines in cost effectiveness acceptability frontier depict strategies with highest expected net monetary benefit dependent on willingness to pay for health benefits 